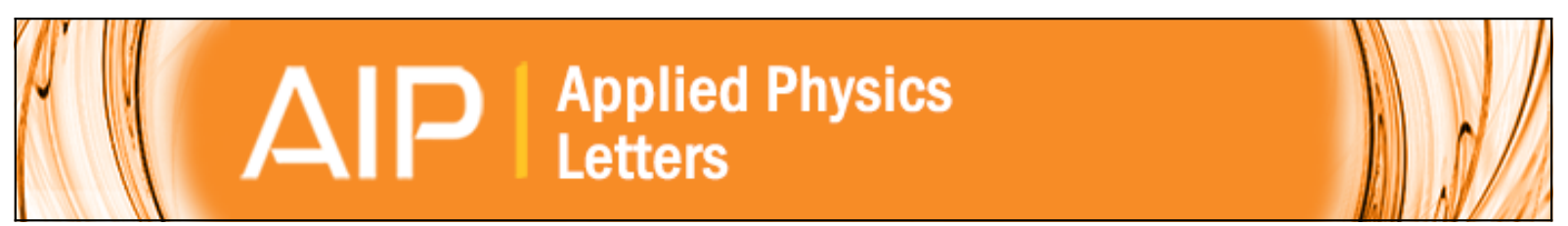

\title{
Beam-induced graphitic carbon cage transformation from sumanene aggregates
}

Jun-ichi Fujita, Masashi Tachi, Katsuhisa Murakami, Hidehiro Sakurai, Yuki Morita, Shuhei Higashibayashi, and Masaki Takeguchi

Citation: Applied Physics Letters 104, 043107 (2014); doi: 10.1063/1.4863739

View online: http://dx.doi.org/10.1063/1.4863739

View Table of Contents: http://scitation.aip.org/content/aip/journal/apl/104/4?ver=pdfcov

Published by the AIP Publishing

$\underset{\substack{\text { Aubsolung } \\ \text { sing }}}{A}$ Re-register for Table of Content Alerts 


\title{
Beam-induced graphitic carbon cage transformation from sumanene aggregates
}

\author{
Jun-ichi Fujita,,${ }^{1,2, a)}$ Masashi Tachi, ${ }^{1,2}$ Katsuhisa Murakami, ${ }^{1,2}$ Hidehiro Sakurai, ${ }^{3}$ \\ Yuki Morita, ${ }^{3}$ Shuhei Higashibayashi, ${ }^{3}$ and Masaki Takeguchi ${ }^{4}$ \\ ${ }^{1}$ Institute of Applied Physics, University of Tsukuba, Tsukuba 305-8573, Japan \\ ${ }^{2}$ Tsukuba Research Center for Interdisciplinary Materials Science (TIMS), Tsukuba 305-8573, Japan \\ ${ }^{3}$ Research Center of Integrative Molecular Systems, Institute for Molecular Science, Myodaiji, \\ Okazaki 444-8787, Japan \\ ${ }^{4}$ Transmission Electron Microscopy Station, National Institute for Materials Science, Tsukuba 305-0047, \\ Japan
}

(Received 28 November 2013; accepted 15 January 2014; published online 29 January 2014)

\begin{abstract}
We found that electron-beam irradiation of sumanene aggregates strongly enhanced their transformation into a graphitic carbon cage, having a diameter of about $20 \mathrm{~nm}$. The threshold electron dose was about $32 \mathrm{mC} / \mathrm{cm}^{2}$ at $200 \mathrm{keV}$, but the transformation is still induced at $20 \mathrm{keV}$. The transformation sequence suggested that the cage was constructed accompanied by the dynamical movement of the transiently linked sumanene molecules in order to pile up inside the shell. Thus, bond excitation in the sumanene molecules rather than a knock-on of carbon atoms seems to be the main cause of the cage transformation. (C) 2014 AIP Publishing LLC.

[http://dx.doi.org/10.1063/1.4863739]
\end{abstract}

The spherically carved surfaces of $\pi$-conjugated structures such as fullerenes and related carbon cages effectively reduce the $\pi$-electron density of the outer surface. ${ }^{1,2}$ The charge transfer from an adopted functional group can thus lead to fullerene derivatives exhibiting useful n-type conducting properties. A notable example is phenyl C61-butyric acid methyl ester (PCBM) that has become an essential material for organic semiconducting device applications. ${ }^{3-5}$ In addition, charge transfer from an encapsulated metal to the outer surface strongly induces an electrical polarization in metal-encapsulated fullerene, which could turn out to be a key mechanism for molecular switching devices. ${ }^{6,7}$ Thus, rational control of these carbon-cage structures ${ }^{8-13}$ would be of great benefit for future electronic applications as well as for nanomechanical applications. ${ }^{14,15}$

Fullerenes and carbon cages have previously been synthesized from a graphite source material at high temperatures of about $3000 \mathrm{~K}^{16,17}$ under the non-equilibrium ambient of Arc-plasma. This transformation of graphite into fullerenes ${ }^{18,19}$ is a well-known technique for the mass production of fullerene and its allotropes ${ }^{20,21}$ including carbon-cages constructed from a large number of carbon atoms. Here, the bottom-up processes based on the so-called "pentagon road"22,23 and "ring coalescence" 24,25 would be the presumed routes to explain the carbon-cage formation mechanism, where very small clusters of carbon atoms coalesce to form fullerene cages. While recent progress in these synthesis methods have remarkably improved the production efficiency, the production mechanism was extrapolated from indirect information of the analyzed species through massspectroscopy and control of the cage structure still requires further understanding of the formation mechanism.

\footnotetext{
${ }^{\text {a) }}$ Author to whom correspondence should be addressed. Electronic mail: fujita@bk.tsukuba.ac.jp. Tel.: +81-29-853-5302.
}

In contrast, in-situ imaging through transmission electron microscopy (TEM) can provide direct evidence for the structural transformation of carbon cages, ${ }^{13,26-28}$ while the significant damage $\mathrm{e}^{29,30}$ to the honeycomb lattice due to so-called "knock-on damage" would be taken into account in the transformation. In particular, the knock-on damage was found to be pronounced when the beam energy was higher than a threshold of about $80 \mathrm{keV},{ }^{31-33}$ and a local bond excitation on the honeycomb lattice also coexisted with the beam irradiation even though the beam acceleration was lower than $1 \mathrm{keV} .^{34}$

Despite the occurrence of beam-induced damage during TEM observation, captured images clarified the basis of the cage-transformation mechanism: static Joule heating on a few layers of graphene (FLG) strongly activated the movement of graphene edges with the assistance of the electron irradiation, ${ }^{35-39}$ where the graphene edges dynamically combine, zipping up with the nearest stack of graphene layers. In addition, the edges of small graphene flakes on graphite begin to curl up under the influence of e-beam irradiation, finally resulting in fullerene formation so as to enclose the curling edges.

In another method, the induction of a cyclic current pulse of about $20 \mathrm{kHz}$ on a graphene edge leading to periodic Joule heating is known to produce various sizes of fullerenes triggered by a cyclic thermal stress, ${ }^{13}$ where the presence of amorphous carbon source material was crucial to promote the cage transformation. However, the repetition of thermal stress produced a larger carbon cage than for that of fullerene. Thus, the final size of produced carbon cages seemed strongly correlated with the initial size of the graphene and/or graphite fragment. The balance between the van der Waals attraction force to the basal plane and the stress to sustain the curling deformation would be the critical factor in determining the cage size.

In contrast to these carbon-cage synthesis methods based on a modification of a flat honeycomb lattice, we 
demonstrated a carbon-cage transformation by assembling a bowl-shaped molecule of sumanene ${ }^{40,41}$ with the assistance of an e-beam-induced reaction, where the decomposition of the sumanene molecules coincided with the rearrangement of molecular fragments into a closed carbon cage. Here, we report on the details of this transformation process and clarify its mechanism using in-situ TEM and scanning electron microscopy (SEM) observations.

Sumanene $\mathrm{S}^{40,41}$ is a bowl-shaped molecule having a chemical composition of $\mathrm{C}_{21} \mathrm{H}_{12}$, as shown in Figure 1(a). Its diameter is about $0.6 \mathrm{~nm}$, and the depth of the bowl is around $0.11 \mathrm{~nm}$. The bowl-shaped molecule easily forms a nanocrystalline aggregate, ${ }^{2,42,43}$ where the crystal structure has the stack of bowl plane aligned along the $\mathrm{C}$-axis having an inter-layer distance of $0.39 \mathrm{~nm}$, and a triangular lattice is created in the C-plane as shown in Fig. 1(a).

We prepared the sumanene nanocrystal sample using the co-precipitation method. The $100 \mu \mathrm{l}$ of tetrahydrofuran solution of sumanene at a concentration of $1 \mathrm{mM}$ was quickly injected into $10 \mathrm{ml}$ of deionized (DI) water. ${ }^{43}$ At this stage, vigorous stirring of the DI-water is the key to obtain a fine and uniform dispersion of the nanocrystal. The dispersed liquid was dropped onto a carbon membrane that was supported on a $\mathrm{Cu}-\mathrm{mesh}$, and the membrane was dried in air at room temperature. A portion of the dispersed sumanene on the carbon membrane tended to form cylindrical crystals as shown in the SEM image and the TEM image in Figs. 1(b) and 1(c), respectively, but the remaining sumanene formed amorphouslike blurred blotches as shown in the SEM image. It should be noted that the secondary electrons emitted from the specimen surface enhanced the image contrast for SEM observation; however, the high-energy electron beam in TEM could only project the image of the sumanene crystal, resulting in an almost transparent background for the amorphous-like sumanene adhesions.

The beam-induced cage transformation was demonstrated using a TEM (JEOL JEM9200) system. We also prepared a sumanene nanocrystal specimen on Si substrate for imaging the low-acceleration e-beam irradiation in a conventional SEM system (Hitachi S4800). Here, a typical beam
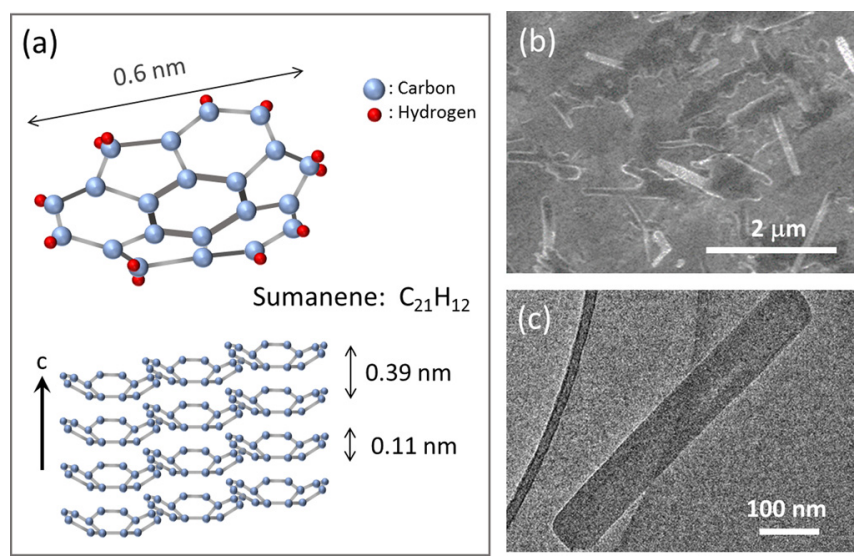

FIG. 1. (a) Bowl-shaped sumanene molecule having a diameter of $0.6 \mathrm{~nm}$ and a depth of $0.11 \mathrm{~nm}$, which is almost the same size as a sliced fullerene. The sumanene molecule constructed a triangular lattice along the c-plane of the crystal. (b) SEM and (c) TEM image of sumanene nanocrystals. Sumanene nanocrystals tended to form cylindrical shapes having lengths from several hundred nanometers to a micrometer. current of about $3.2 \mathrm{nA}$ was used for TEM observation under a back pressure of about $2 \times 10^{-5} \mathrm{~Pa}$, and $33 \mathrm{pA}$ for SEM observation under a back pressure of about $2 \times 10^{-4} \mathrm{~Pa}$.

Figure 2 shows the typical cage-transformation sequence that was recorded during TEM observation. Sumanene molecules dispersed on a carbon membrane tended to form an amorphous-like aggregation that can be observed as blurry blotches after a relative long beam-irradiation time of about $30 \mathrm{~min}$, corresponding to an electron dose of about 32 $\mathrm{mC} / \mathrm{cm}^{2}$, where we first noticed this weak change in the contrast during the adjustment process of the e-beam optics. The subsequent change in the morphology following by the cage transformation rapidly progressed within a minute as follows: The first step was the appearance of a relatively dark-contrast area in the blurred blotch, with its boundary indicated by the dashed line in Fig. 2(a). Then, a distinctive spherical condensation having a diameter of about $20 \mathrm{~nm}$ soon appeared in the middle of the blotch. The shape of the dark spherical condensation continuously and quickly changed to reveal a clear outer fringe containing a certain amount of fragments in the core region within $10 \mathrm{~s}$, as shown in Fig. 2(b). The internal fragments moved dynamically and seemed to feed into the shell wall from inside the shell, enlarging the partial hollow space inside the shell. These encapsulated fragments, which would be transiently cross-linked sumanene molecules, gradually disappeared accompanying by dynamical movement inside the cage as shown in Fig. 2(c). Finally, the fragments seemed to be completely absorbed, and the enlarged shell
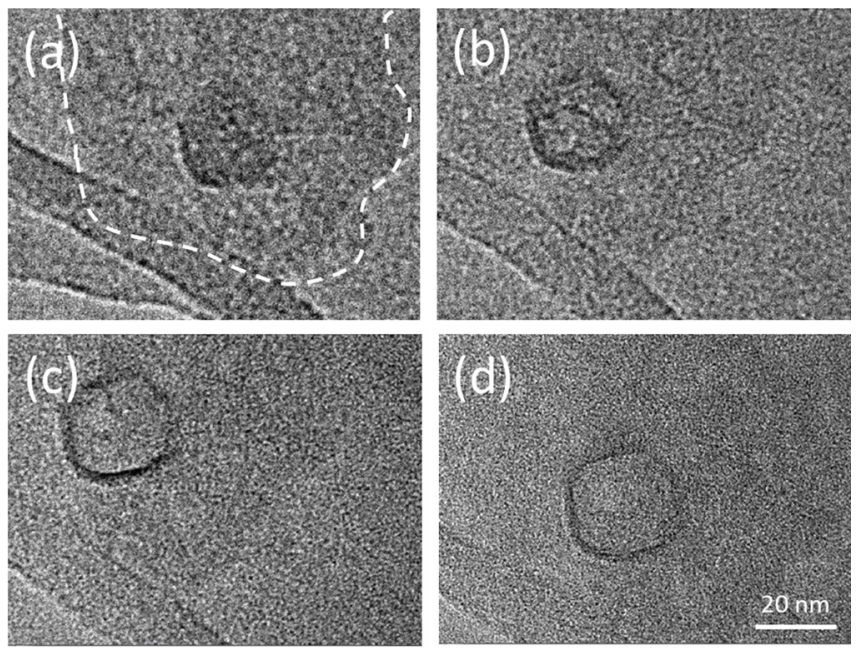

(e)

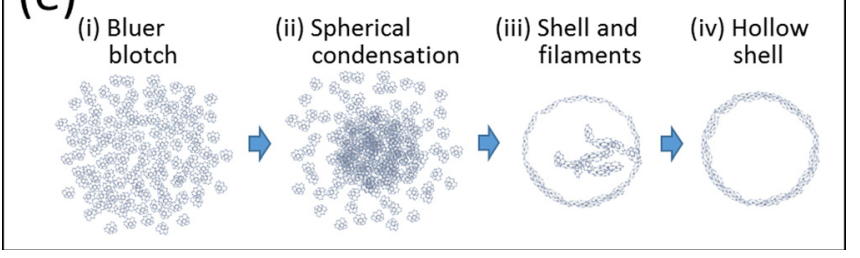

FIG. 2. Sequence of the cage formation observed under TEM imaging field. The accumulated electron dose for the specimen was about $32 \mathrm{mC} / \mathrm{cm}^{2}$ during a system adjustment time of $30 \mathrm{~min}$. The sequence is describes as (a) spherical condensation; (b) condensed molecules create a hollow space containing some inner fragments after $10 \mathrm{~s}$ of beam irradiation; (c) contrast of the outer shell and the inside space become clear; (d) cage transformation complete. (e) Schematic illustration of the cage transformation (Multimedia view). [URL: http://dx.doi.org/10.1063/1.4863739.1]. 
produced a fully hollow space inside the carbon cage having a diameter of about $25 \mathrm{~nm}$, as shown in Fig. 2(d).

Many of these beam-induced carbon-cage transformations occurred reproducibility as shown in (a) to (e) of Fig. 3. The produced cage sizes varied from 10 to $40 \mathrm{~nm}$, but the size distribution seemed to have a strong correlation with the initial size of the sumanene aggregate on the carbon membrane. Many of the transformed carbon cages have a spherical shape and are composed of a single layer to two or three layers of shells, while some were composed of angular shells (Fig. 3(c)).

Such a cage structure created on a carbon membrane can be projected onto a TEM screen and/or imaging device as a dark ring when the beam optics are adjusted to be slightly defocused because the distinctive diffraction of the primary electron is induced at the graphitic plane parallel to the azimuth of the incident beam. Therefore, the electron that passed through the rounded top surface of the cage created an internal bright spot and a monotonic area as shown in Figs. 3(a)-3(e). However, the SEM image in Fig. 3(f), which was taken at the same location as the TEM image in Fig. 3(e), clearly suggests that the ring-shaped contrast in the TEM image exactly represents the three-dimensional cage structure.

We also investigated a similar beam-induced transformation under the relatively low acceleration beam energy of the SEM system. Here, the dispersion of sumanene was dropped onto a heated $\mathrm{SiO}_{2}$ at $80{ }^{\circ} \mathrm{C}$. The surface of $\mathrm{SiO}_{2}$ was oxygen-plasma treated in order to improve the wettability, and the liquid was then uniformly dispersed over the
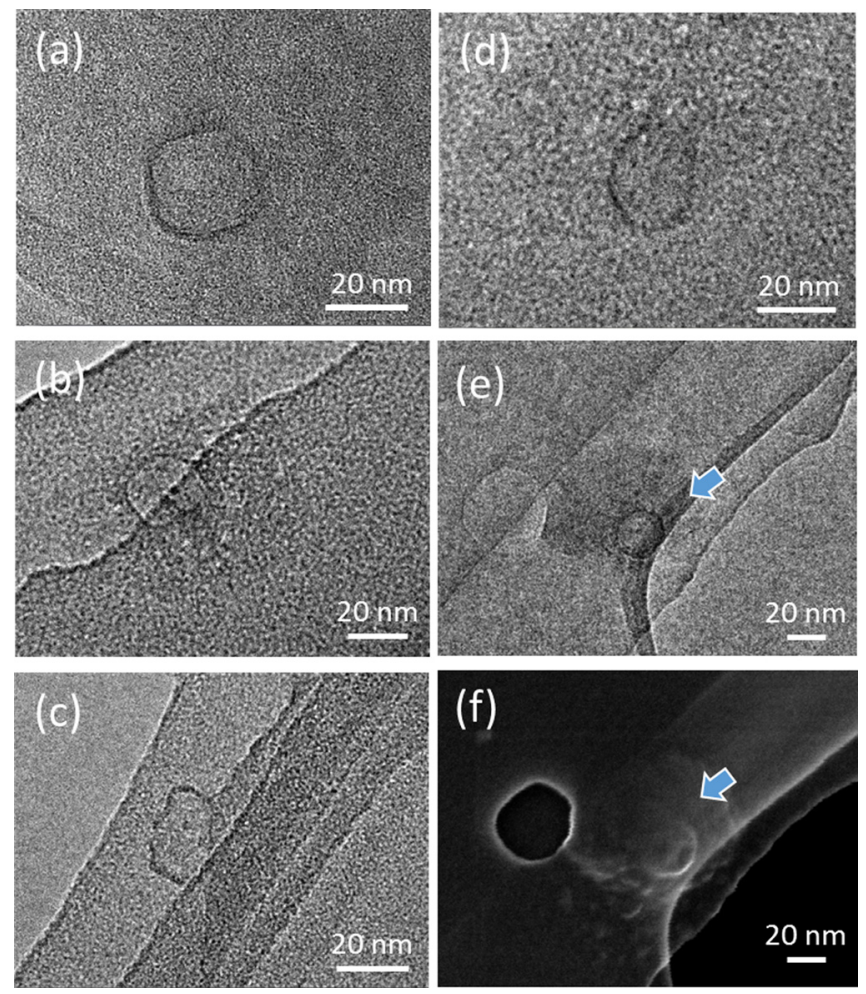

FIG. 3. Typical carbon cages that were created on a carbon membrane from sumanene aggregates. While the sizes and shapes were different, it should be noted that all of the carbon cages were created on a blurred blotch of sumanene. The SEM photograph in (f) corresponds to the TEM image in (e), where the ring-shaped structure indicated by the arrow exactly corresponds to the three-dimensional bubble. entire surface. The heated $\mathrm{SiO}_{2}$ surface soon vaporized the DI-water and sumanene nanocrystals were produced, which tended to have a rectangular shape and a width of several hundred nanometers.

The carbon-cage transformations were also induced at almost the same electron dose of around $24 \mathrm{mC} / \mathrm{cm}^{2}$ at $20 \mathrm{keV}$. A typical cage-production sequence is shown in Figure 4, where the gray-colored sumanene crystal forms a rectangular-shaped thin crystal on the $\mathrm{SiO}_{2}$ substrate. The SEM images were constructed from the secondary electrons from the beam irradiation point and the existence of steep protrusions and/or spherical nanostructure strongly enhanced the emission of secondary electrons. Therefore, the carbon cage should appear as a bright spot in the SEM image. While no bright spots could be detected at the initial SEM frame scanning, after electron irradiation of $24 \mathrm{mC} / \mathrm{cm}^{2}$, a small but distinguishable bright spot appeared, which is indicated by the white arrow in Fig. 4(b). The bright spot grew larger while accumulating an electron dose of $48 \mathrm{mC} / \mathrm{cm}^{2}$ (Fig. 4(c)) and $64 \mathrm{mC} / \mathrm{cm}^{2}$ (Fig. 4(d)). The clear contrast of the bright spots shows exactly an ordered nanostructure protruding from the crystal surface that could be confirmed in the tilted SEM images of Figs. 4(e) and 4(f). Here, the observing direction is indicated by the dashed arrow in Fig. 4(d).

We confirmed that the bright spots appearing on the sumanene aggregates were the same carbon cages as those produced by the electron beam irradiation at $200 \mathrm{keV}$, as shown in Fig. 5. Here, an amorphous carbon membrane was utilized as the substrate for the convenience of TEM observation, but some clear white spots appeared on the surface as shown in Fig. 5(a) after the electron beam irradiation at
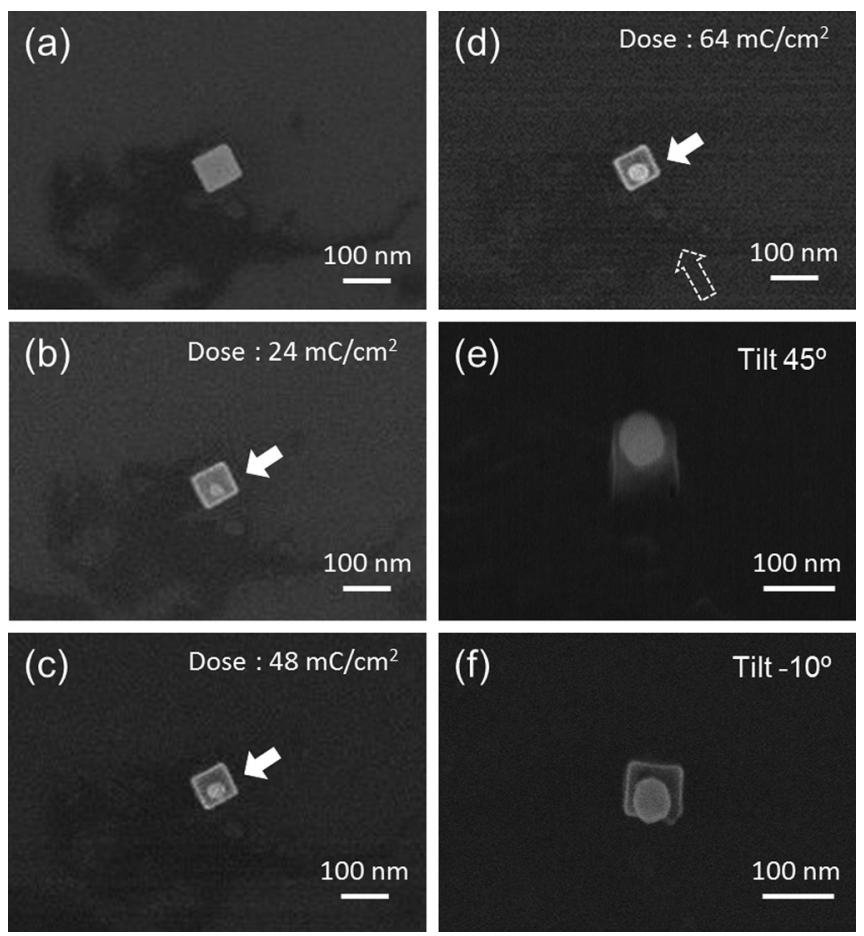

FIG. 4. Typical cage-production sequences on sumanene nanocrystals under electron-beam irradiation at $20 \mathrm{keV}$ : (a) the initial state before electron irradiation; (b) a bright spot appears on a rectangular crystal surface (indicated by the arrow) at a dose of $24 \mathrm{mC} / \mathrm{cm}^{2}$; the spot grew larger as the dose was increased to (c) $48 \mathrm{mC} / \mathrm{cm}^{2}$ and (d) $64 \mathrm{mC} / \mathrm{cm}^{2}$. 

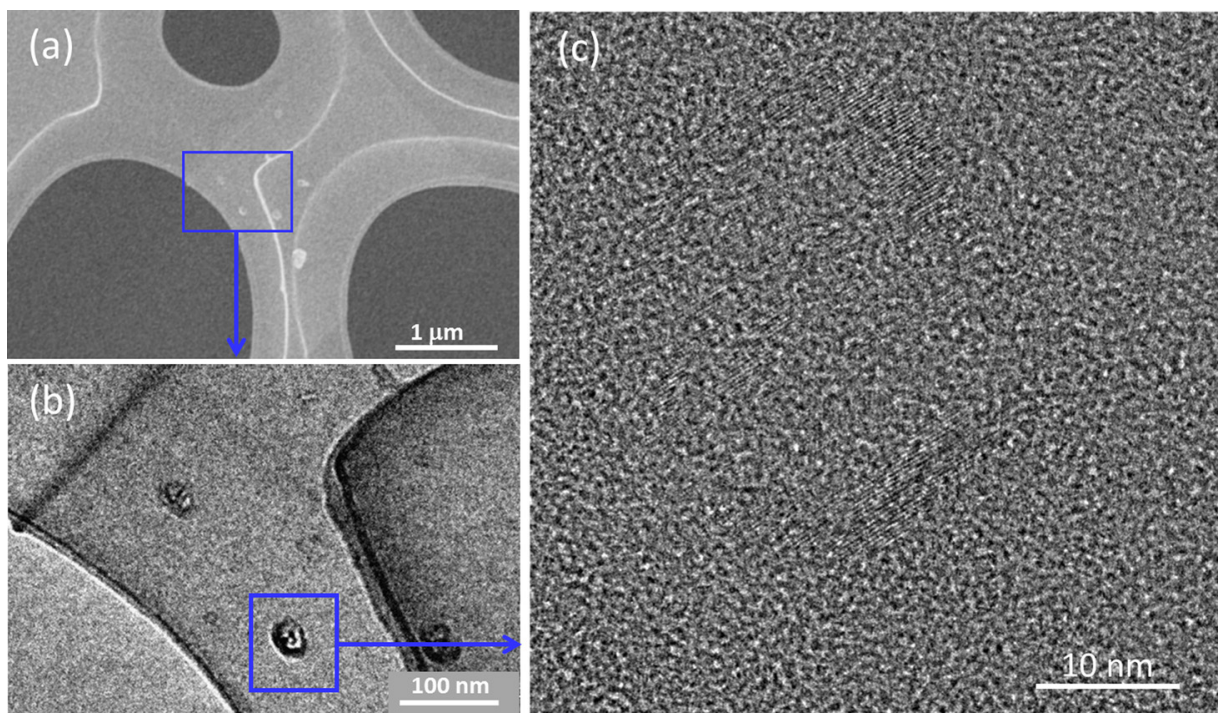

FIG. 5. (a) SEM image of a beamirradiated carbon membrane with some bright spots appearing at the center. The rectangular area that was rotated about $45^{\circ}$ in the counterclockwise direction was the beam-irradiated area. (b) Bright spots created by electron irradiation at $20 \mathrm{keV}$ were basically the same carbon cages created by electron beam irradiation at $200 \mathrm{keV}$ under the TEM environment. (c) This HR-TEM image suggests that the cage was constructed with a graphitic lattice structure.

$20 \mathrm{keV}$. The TEM image of Fig. 5(b) clearly suggested that the white spot is a carbon cage that was essentially the same one that was synthesized with the electron-beam irradiation at $200 \mathrm{keV}$ under the TEM environment. The highresolution (HR) TEM image in Fig. 5(c) also suggests that the cage was constructed with a graphitic surface, but it was partially destructed. Because the intense irradiation of HRTEM electron beam easily damaged the cage structure, the HR-TEM image shows the intermittent structure during the destructing process.

It should be noted that the carbon-cage transformation of sumanene can be induced at $20 \mathrm{keV}$, where the "knockon" of carbon atoms could not be induced; rather, the desorption of hydrogen atoms simultaneous with the bond excitation of five- and/or six-membered rings would be induced. Thus, we believe that bond excitation of the sumanene molecule followed by the cross-linking of sumanene molecules is the main cause for the carbon-cage transformations in this beam-induced reaction.

While the threshold electron dose shows a slight difference between the beam energies of TEM and SEM, in terms of the size distribution of the cages, the diameter of many of the cages converge around $20 \mathrm{~nm}$, as shown in the histogram of Figure 6. The size distribution may give crucial information

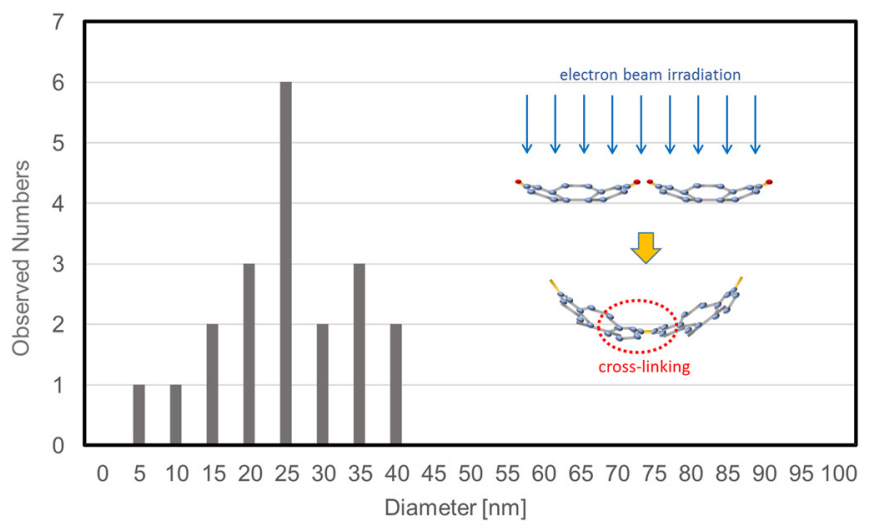

FIG. 6. Histogram of the size of created carbon cages, which were distributed from $5-40 \mathrm{~nm}$. to understand the cage-transformation mechanism under electron beam irradiation. The sumanene is a small bowl-shaped molecule having a diameter of about $0.6 \mathrm{~nm}$; thus, the simplest assembly of the sumanene molecule would create a carbon cage almost the same size as fullerene.

However, electron-beam irradiation can break the bonds of the pentagonal- and hexagonal-membered rings in the sumanene as well as dissociate the hydrogen even though the irradiated beam energy of $20 \mathrm{keV}$ is lower than the knock-on threshold. $^{44,45}$ The internal strain at the pentagonalmembered ring should hold a strong strain in order to create the steep curvature of fullerene; thus, the destructed sumanene would have a much gentler bowl curvature. The dangling bonds after the dissociation of hydrogen can participate in the cross-linking of sumanene molecules, resulting in the assembly of such relaxed sumanene molecules and the production of larger carbon cages having a size several tens of nanometers, much larger than that of fullerene.

The knock-on of carbon atoms at the edge of a small graphene fragment was the intrinsic mechanism of the fullerene transformation from graphite under high-energy electron irradiation; however, the cage transformation from sumanene seemed to be a result of the beam-induced reaction of both bond scissoring and cross-linking of the sumanene molecule.

In summary, we found that the electron-beam irradiation of sumanene molecules effectively induced their transformation into graphitic carbon cages, where the cages were constructed from 1-3 graphitic shell layers. The threshold electron dose was typically around $32 \mathrm{mC} / \mathrm{cm}^{2}$ at a beam energy of $200 \mathrm{keV}$, but the transformation is still induced even when the beam energy was reduced to $20 \mathrm{keV}$, where the threshold dose was reduced to about $24 \mathrm{mC} / \mathrm{cm}^{2}$. This transformation from sumanene molecules is in contrast to fullerene formation at graphene edges under electron-beam irradiation, where the defects remaining after knocked-on carbon atoms induces the curing. However, the transformation of sumanene molecules into carbon cages seemed to be dominated by bond excitation in the molecule in connection with the opening of the pentagonal-membered rings and cross-linking of the molecules. Thus, the transformed graphitic carbon cages assembled from relaxed sumanene 
molecules have a large diameter of about $20 \mathrm{~nm}$ compared to that of fullerene, and the diameter of the carbon cage would be automatically determined to be around $20 \mathrm{~nm}$.

Finally, the gentle reaction and the deformation process would allow for the incorporation of metal species into the cage, combining with the adsorption of metal-organic molecules. While further study is merited to gain a detailed understanding of the cage-transformation mechanism, we believe that this bottom-up process, based on the beam-induced sumanene molecule reaction, would be a promising new method to produce functional carbon cages for future electronic device applications.

This work was supported by JSPS KAKENHI Grant No. 23246063.

${ }^{1}$ R. C. Haddon, R. E. Palmer, H. W. Kroto, and P. A. Sermon, Philos. Trans. R. Soc., A 343, 53 (1993).

${ }^{2}$ S. Mebs, M. Weber, P. Luger, B. M. Schmidt, H. Sakurai, S. Higashibayashi, S. Onogi, and D. Lentz, Org. Biomol. Chem. 10, 2218 (2012).

${ }^{3}$ D. M. Guldi, B. M. Illescas, C. M. Atienza, M. Wielopolskia, and N. Martín, Chem. Soc. Rev. 38, 1587 (2009).

${ }^{4}$ Y. Maeyoshi, A. Saeki, S. Suwa, M. Omichi, H. Marui, A. Asano, S. Tsukuda, M. Sugimoto, A. Kishimura, K. Kataoka, and S. Seki, Sci. Rep. 2, 600 (2012).

${ }^{5}$ E. Lörtscher, V. Geskin, B. Gotsmann, J. Fock, J. K. Sørensen, T. Bjørnholm, J. Cornil, H. S. J. van der Zant, and H. Riel, Small 9, 209 (2013).

${ }^{6}$ L. Liu, S. Liu, X. Chen, C. Li, J. Ling, X. Liu, Y. Cai, and L. Wang, Sci. Rep. 3, 3062 (2013).

${ }^{7}$ S. Vijayaraghavan, D. Écija, W. Auwarter, S. Joshi, K. Seufert, A. P. Seitsonen, K. Tashiro, and J. V. Barth, Nano Lett. 12, 4077 (2012).

${ }^{8}$ A. V. Krasheninnikov and F. Banhart, Nature Mater. 6, 723 (2007).

${ }^{9}$ F. Banhart, Rep. Prog. Phys. 62, 1181 (1999).

${ }^{10}$ C. Kiang, W. A. Goddard III, R. Beyers, and D. S. Bethune, J. Phys. Chem. 100, 3749 (1996).

${ }^{11}$ R. Ueki, R. Endo, T. Hikata, S. Okubo, R. Utsunomiya, and J. Fujita, Microelectron. Eng. 88, 2516 (2011).

${ }^{12}$ T. Nishijima, R. Ueki, Y. Miyazawa, and J. Fujita, Microelectron. Eng. 88, 2519 (2011).

${ }^{13}$ T. Nishijima, R. Ueki, E. Kano, and J. Fujita, Jpn. J. Appl Phys., Part 1 51, 06FD20 (2012).

${ }^{14}$ C. Lee, X. Wei, J. W. Kysar, and J. Hone: Science 321, 385 (2008).
${ }^{15}$ A. Kis and A. Zettl, Philos. Trans. R. Soc., A 366, 1591 (2008).

${ }^{16}$ R. E. Smalley, Acc. Chem. Res. 25, 98 (1992).

${ }^{17}$ W. Krätschmer, L. D. Lamb, K. Fostiropoulos, and D. R. Huffman, Nature 347, 354 (1990)

${ }^{18}$ H. W. Kroto, J. R. Heath, S. C. O'Brien, R. F. Curl, and R. E. Smalley, Nature 318, 162 (1985).

${ }^{19}$ R. E. Smalley, Rev. Mod. Phys. 69, 723 (1997).

${ }^{20}$ Y. Z. Tan, J. Li, F. Zhu, X. Han, W. S. Jiang, R. B. Huang, Z. Zheng, Z. Z. Qian, R. T. Chen, Z. J. Liao, S. Y. Xie, X. Lu, and L. S. Zheng, Nat. Chem. 2, 269 (2010).

${ }^{21}$ D. Ugarte, Europhys. Lett. 22, 45 (1993).

${ }^{22}$ N. S. Goroff, Acc. Chem. Res. 29, 77 (1996).

${ }^{23}$ H. W. Kroto and K. McKay, Nature 331, 328 (1988).

${ }^{24}$ J. M. Hunter, J. L. Fye, E. J. Roskamp, and M. F. Jarrold, J. Phys. Chem. 98, 1810 (1994).

${ }^{25}$ Y. Rubin, M. Kahr, C. B. Knobler, F. Diederich, and C. L. Wilkins, J. Am. Chem. Soc. 113, 495 (1991).

${ }^{26}$ T. F'tiller and F. Banhart, Chem. Phys. Lett. 254, 372 (1996).

${ }^{27}$ C. Jin, K. Suenaga, and S. Iijima, J. Phys. Chem. C 113, 5043 (2009).

${ }^{28}$ P. J. F. Harris. Carbon 50, 3195 (2012).

${ }^{29}$ M. Takeuchi, S. Muto, T. Tanabe, S. Arai, and T. Kuroyanagi, Philos. Mag. 76, 691 (1997).

${ }^{30}$ K. Molhave, S. B. Gudnason, A. T. Pedersen, C. H. Clausen, A. Horsewell, and P. Bggild, Ultramiocroscopy 108, 52 (2007).

${ }^{31}$ B. W. Smith and D. E. Luzzia, J. Appl. Phys. 90, 3509 (2001).

${ }^{32}$ A. Zobelli, A. Gloter, C. P. Ewels, G. Seifert, and C. Colliex, Phys. Rev. B 75, 245402 (2007).

${ }^{33}$ J. H. Warner, M. H. Rummeli, L. Ge, T. Gemming, B. Montanari, N. M. Harrison, B. Buchner, and G. A. D. Briggs, Nat. Nanotechnol. 4, 500 (2009).

${ }^{34}$ S. Suzuki and Y. Kobayashi, J. Phys. Chem. C 111, 4524 (2007).

${ }^{35}$ D. Ugarte, Nature 359, 707 (1992).

${ }^{36}$ J. Y. Huang, Phys. Rev. Lett. 99, 175503 (2007).

${ }^{37}$ J. Y. Huang, F. Ding, B. I. Yakobson, P. Lu, L. Qi, and J. Li, Proc. Natl. Acad. Sci. U.S.A. 106, 10103 (2009).

${ }^{38}$ X. Jia, M. Hofmann, V. Meunier, B. G. Sumpter, J. C. Delgado, J. M. Romo-Herrera, H. Son, Y. P. Hsieh, A. Reina, J. Kong, M. Terrones, and M. S. Dresselhaus, Science 323, 1701 (2009).

${ }^{39}$ A. Chuvilin, U. Kaiser, E. Bichoutskaia, N. A. Besley, and A. N. Khlobystov, Nat. Chem. 2, 450 (2010).

${ }^{40}$ H. Sakurai, T. Daiko, and T. Hirao, Science 301, 1878 (2003).

${ }^{41}$ S. Higashibayashi and H. Sakurai, Chem. Lett. 40, 122 (2011).

${ }^{42}$ H. Sakurai, T. Daiko, H. Sakane, T. Amaya, and T. Hirao, J. Am. Chem. Soc. 127, 11580 (2005).

${ }^{43}$ Y. Morita, S. Nakao, S. Haesuwannakij, S. Higashibayashi, and $\mathrm{H}$. Sakurai, Chem. Commun. 48, 9050 (2012).

${ }^{44}$ F. Banhart, J. Mater Sci. 41, 4505 (2006).

${ }^{45}$ W. L. Fite and R. T. Brackmann, Phys. Rev. 112, 1151 (1958). 